\title{
Benchmarking of Problems and Solvers: a Game-Theoretic Approach
}

\author{
Joseph Gogodze*
}

\begin{abstract}
In this note, we propose a game-theoretic approach for benchmarking computational problems and their solvers. The approach takes an assessment matrix as a payoff matrix for some zero-sum matrix game in which the first player chooses a problem and the second player chooses a solver. The solution in mixed strategies of this game is used to construct a notionally objective ranking of the problems and solvers under consideration. The proposed approach is illustrated in terms of an example to demonstrate its viability and its suitability for applications.
\end{abstract}

Keywords: benchmarking, software, solvers, problems, testing, multi objective decisionmaking problem

\section{Introduction}

In recent years, evaluating the performance of solvers has become a topic of intense study, and various approaches have been discussed in the literature. Most benchmarking tests produce tables showing each solver's performance for each problem according to a specified evaluation metric, such as the central processing unit (CPU) time, number of function evaluations, or number of iterations. Interpretations of this data, i.e., the selection of benchmarking method, currently depends on the subjective tastes and preferences of individual researchers, who evaluate solvers using different problem sets and metrics, [2$5,7,8,10,13,14,20]$.

* Institute of Control System, TECHINFORMI, Georgian Technical University, 77 Kostava str., ,0175 Tbilisi, Georgia. Email: sosogogodze@gtu.ge 
This study introduces a new benchmarking approach that explores the natural relations between problems and solvers as determined by their evaluation tables. Specifically, we present data for benchmarking in the form of a benchmarking context, i.e., as a triple $\langle S, P, J\rangle$ where $S$ and $P$ are sets of solvers and problems, respectively and $J: S \times P \rightarrow \mathbb{R}$ is an assessment function (a performance metric or evaluation metric). This concept is quite general and emphasizes that problem and solver benchmarking cannot be considered independently. Throughout the paper the benchmarking context $\langle S, P, J\rangle$ assumes that the sets of solvers and problems are finite.

The benchmarking procedure proposed in this study uses the data encapsulated by the given benchmarking context $\langle S, P, J\rangle$ and a new multi-objective decision making (MODM) procedure. Specifically, we consider the set of problems as a set of alternatives and the set of solvers as a set of criteria. Now, we can define a decision matrix as a matrix whose elements exhibit the performance of different alternatives (i.e., solvers) with respect to various criteria (i.e., problems) through the assessment function.

The rationale of such consideration is that such a multi-objective formulation allows us to use the concept of Pareto optimality and a vast arsenal of different approaches for Pareto optimization. However, it must be stressed that a characteristic feature of Pareto optimality is that the set of Pareto-optimal alternatives is large and that all Pareto-optimal alternatives must be considered as mathematically equal. On the other hand, because decisions are usually expected to be unique, additional factors are considered for selecting specific and/or in some sense more appropriate alternatives from the set of Pareto-optimal alternatives. Our approach allows us to select, in some sense, appropriate solvers. The essence of the method lies in the fact that an (in some sense) objective weighting method for the MODM problem described above can be obtained solving a special two-person zero-sum game. Specifically, we can consider the decision matrix described above as a pay-off matrix for some zero-sum matrix game in which the S-player chooses one of the solvers (i.e., alternatives) from $S$ and the Pplayer chooses one of the problems (i.e., criteria) from $P$. Solving this game using mixed strategies, we can find both appropriate solvers and reasonable rankings of solvers and problems at the same time.

We demonstrate the possibilities of the proposed method on a concrete example. Specifically, as an illustrative example, based on data from [16], we benchmark 9 differential evolutionary algorithms on a set of 50 test problems, in accordance with the ERT RSE (Random Sampling Equivalent Expected Run Time) performance metric.

The remainder of this paper is organized as follows. Section 2 describes the proposed methodology for evaluating and comparing solvers and problems. Section 3 considers the applications of the proposed tool in a selected benchmarking problem. Section 4 draws conclusions.

\section{Proposed Methods}

Throughout the paper, $\mathbb{R}^{n}$ is $n$-dimensional space, with norm $\|\cdot\|$ and scalar product $\langle\cdot$,$\rangle . Moreover, we use the following notations for the special sets and special vectors:$ 


$$
\begin{gathered}
\mathbb{R}_{+}^{n}=\left\{\xi \in \mathbb{R}^{n} \mid \xi_{k} \geq 0, k=1, \ldots, n\right\}, \Delta_{n}=\left\{\xi \in \mathbb{R}_{+}^{n} \mid \sum_{k=1}^{n} \xi_{k}=1\right\}, \\
e_{k}=\left(0, \ldots, 1_{(k)}, \ldots, 0\right) \in \mathbb{R}^{n}, k=1, \ldots, n ; 1_{n}=(1, \ldots, 1) \in \mathbb{R}^{n} ; 0_{n}=(0, \ldots, 0) \in \mathbb{R}^{n}
\end{gathered}
$$

\subsection{Preliminaries regarding MODM problems}

We begin here with notations and definitions that are necessary for our further considerations. We assume that the initial data for decision-making are presented as a decision matrix, $X$, the elements of which exhibit the performance of diverse alternatives with respect to various criteria:

$$
X=\left[\begin{array}{ccc}
x_{11} & \cdots & x_{1 n} \\
\vdots & \ddots & \vdots \\
x_{m 1} & \cdots & x_{m n}
\end{array}\right]
$$

where $x_{i j}$ the performance is measure of the $i$-th alternative on the $j$-th criterion $\quad(i=$ $1, \ldots n, j=1, \ldots, n), m$ is the number of alternatives, and $n$ is the number of criteria.

For simplicity, we assume further that each criterion can be treated as non-beneficial (for which lower values are preferable) criteria. We assume in addition that the decision matrix is normalized for ensuring the comparability of its elements and is such that $\sum_{k=1}^{n} x_{k j}>0$, $x_{j}^{\min }=\min \left\{x_{1 j}, \ldots, x_{m j}\right\}=0, \quad x_{j}^{\max }=\max \left\{x_{1 j}, \ldots, x_{m j}\right\}=1 \quad$ for $\quad$ all $j=1, \ldots, \mathrm{n}$ i.e. elements of the matrix $X$ are dimensionless numbers representing the normalized performance of the $i$-th alternative on the $j$-th criterion $(i=1, \ldots n, j=1, \ldots, n)$. Thus, the decision-making procedure's goal after the normalization procedure is simultaneous minimization of all criteria, i.e., we obtain a typical multi-objective optimization problem.

Recall now the basic concepts of multi-objective optimization theory. To this end we introduce the following notations: alternatives will be denote by $a_{i}, i=1, \ldots, m, \quad A=$ $\left\{a_{1}, \ldots, a_{m}\right\}$ and criteria by $c_{j}: A \rightarrow \mathbb{R}, j=1, \ldots, n$ with the result that $x_{i j}=c_{j}\left(a_{i}\right), i=$ $1, \ldots m, j=1, \ldots, n$. Further, the set $A$ will be designated as the set of alternatives, map $\vec{c}=\left(c_{1}, \ldots, c_{n}\right)$ will be designated as the criterion map (correspondingly $c_{j}$ is the $j$-th objective $j=1, \ldots, \mathrm{n}$ ), and the set $\vec{c}(A)$ will be designated as the set of admissible criterion values. The following concepts are also associated with the criterion map and the set of alternatives. We say that an alternative $\bar{a} \in A$ is a minimizer of the $j$-th criterion, if $c_{j}(\bar{a})=$ $\min _{a \in A} c_{j}(a)$. We denote by $A_{\min }^{j}(\vec{c})$ the set of all minimizers of the $j$-th objective, $j=1, \ldots, \mathrm{n}$. Correspondingly, a point $\xi_{j}=\vec{c}(a) \in \vec{c}(A)$, where $a \in A_{\text {min }}^{j}(\vec{c})$ will be designated as an anchor point, $j=1, \ldots, \mathrm{n}$. The point $\xi^{I}=\left(\xi_{1}^{I}, \ldots, \xi_{n}^{I}\right)$ where if $\xi_{j}^{I}=\min _{a \in A} c_{j}(a)$, objective $j=1, \ldots, \mathrm{n}$, will be designated as an ideal point. We say that an ideal point is attainable if there exists an alternative $a^{I} \in A$ such that $\xi^{I}=\vec{c}\left(a^{I}\right)$.

We say that point $a_{*} \in A$ is Pareto-optimal (efficient) if there is no $a \in A$ with $c_{j}(a) \leq$ $c_{j}\left(a_{*}\right)$ for all $j=1, \ldots, \mathrm{n}$, and there exists an index $j_{0} \in\{1, \ldots, n\}$ such that $c_{j_{0}}(a)<c_{j_{0}}\left(a_{*}\right)$. The set of all efficient alternatives will be denoted $A_{e}$ and designated as a Pareto set Accordingly, $\vec{c}\left(A_{e}\right)$ will be designated as an efficient front. 
A very common method for solving the MODM problem is the weighting method. This method requires specifying how we are to determine what we consider to be objective weights. To this end, for simplicity we consider here only entropy method (ENT), [22]. To determine objective weights using the entropy measure, the decision matrix must to be normalized as follows:

$$
p_{i j}=x_{i j} / \sum_{k=1}^{m} x_{k j}(i=1, \ldots n, j=1, \ldots, n) .
$$

Now, the amount of decision information contained in the matrix $p_{i j}$ and emitted from each criterion can be measured by the entropy values

$$
e_{j}=-\frac{1}{\ln m} \sum_{k=1}^{m} p_{k j} \ln \left(p_{k j}\right), \quad j=1, \ldots, n .
$$

Correspondingly, the degree of divergence $d_{j}=1-e_{j}$ can be defined and the objective weight can be calculated as

$$
w_{j}=d_{j} / \sum_{k=1}^{m} d_{k}, j=1, \ldots, n .
$$

It is possible to determine objective weights similarly for alternatives, but we omit the corresponding details here.

\subsection{Game-theoretic approach for solving MODM problems}

Here, we present our game-theoretic approach for solving MODM problems, [11]. The proposed method considers the matrix $X$ as a pay-off matrix for some zero-sum matrix game. We can interpret this game as follows. The row-player ( $A$-player) chooses one of the alternatives $a \in A$, and the column-player ( $C$-player) chooses one of the criteria $c \in C$. The quantity $x_{i j}=c_{j}\left(a_{i}\right)$ is a sum paid to the $A$-player by the $C$-player when the $A$-player chooses the alternative $a_{i} \in A$, and the $C$-player chooses the criteria $c_{j} \in C, i=1, \ldots m, j=$ $1, \ldots, n$. The mixed strategy for the $A$-player is a vector $\xi \in \Delta_{m}$, and the mixed strategy for the $C$-player is a vector $\zeta \in \Delta_{n}$. Correspondingly, components $\xi_{k}, k=1, \ldots, m,\left(\zeta_{k}, k=\right.$ $1, \ldots, n)$ are the probabilities of choices for alternative $k$ by the $A$-player (resp., criteria $k$ by the $C$-player). Hence, for mixed strategies $\xi \in \Delta_{m}, \zeta \in \Delta_{n}$, the expected pay-off for the $A$-player is

$$
\Lambda(\xi, \zeta)=\sum_{i=1}^{m} \sum_{j=1}^{n} x_{i j} \xi_{i} \zeta_{j}=\sum_{i=1}^{m} P_{\zeta}\left(a_{i}\right) \xi_{i}=\sum_{j=1}^{n} Q_{\xi}\left(c_{j}\right) \zeta_{i},
$$

where we use the notation

$$
P_{\zeta}(a)=\sum_{j=1}^{n} c_{j}(a), \forall a \in A ; \quad Q_{\xi}(c)=\sum_{i=1}^{m} c\left(a_{i}\right) \forall c \in C .
$$


Note that $P_{\zeta}(a)$ can be interpreted as the expected pay-off of the alternative $a \in A$, when choosing the $C$-player's mixed strategies $\zeta \in \Delta_{n}$ and $Q_{\xi}(c)$ can be interpreted as expected pay-offs for the criterion $c \in C$ when choosing the $A$-player's mixed strategies $\xi \in \Delta_{m}$. Recall also that a pair of mixed strategies $\left(\xi^{*}, \zeta^{*}\right) \in \Delta_{m} \times \Delta_{n}$ is a Nash equilibrium solution of the considered zero-sum matrix game if and only if

$$
\max _{\xi \in \Delta_{m}} \min _{\zeta \in \Delta_{n}} \Lambda(\xi, \zeta)=\min _{\zeta \in \Delta_{n}} \max _{\xi \in \Delta_{m}} \Lambda(\xi, \zeta)=\Lambda\left(\zeta^{*}, \zeta^{*}\right)
$$

Let $\left(\zeta^{*}, \zeta^{*}\right) \in \Delta_{m} \times \Delta_{n}$ be a solution of the considered zero-sum matrix game. Now, we interpret $\zeta^{*} \in \Delta_{n}$ as a properly chosen weight and consider $P_{\xi^{*}}^{\vec{c}}(a)=\sum_{j=1}^{n} c_{j}(a) \zeta_{j}^{*}$ as a true aggregation of performance criteria. Moreover, it is well-known that any solution of this minimization problem

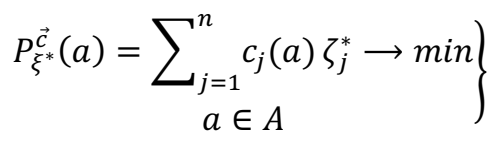

is always Pareto optimal, and hence, the presented approach allows the selection of some Pareto-optimal alternative, which can be considered as appropriate.

The relevant interpretation of the procedure described above is necessary for determining in what sense this obtained Pareto-optimal alternative is appropriate. We assume that the $A$ -player and $C$-player are represented by the populations designated as the $A$-population and $C$-population, respectively. Moreover, we also assume that to each alternative (criterion) there corresponds the subpopulation of individuals that dispose of this and only this alternative (criterion) in the $A$-population (resp., $C$-population), and that such subpopulations cover the entire $A$-population (resp., $C$-population). We also interpret component $\xi_{k}, k=$ $1, \ldots, m,\left(\zeta_{k}, k=1, \ldots, n\right)$ of a mixed strategy $\xi \in \Delta_{m}\left(\zeta \in \Delta_{n}\right)$ as sharing in a corresponding subpopulation in the $A$-population (resp., $C$-population).

It is useful to be able to compare the proposed method,with some known method of MODM-problem solution. For this purpose, we use the ENT method described in the previous subsection. After the weights determining by the ENT method or by the GTR method, the criteria can be aggregated/scalarized into the single criterion. The aggregate criterion's value on an alternative defines rating of this alternative. The subsequent ordering (by ascending or descending ratings-at wish) of the alternatives determines their ENTranking or GTR-ranking, respectively.

\subsection{Benchmarking problem}

Consider a set $P$ of problems, a set $S$ of solvers, and a function $J: S \times P \rightarrow \mathbb{R}$ - the assessment function (performance metric). Further, assume for definiteness that the high and low values of $J$ correspond to the worst and best cases, respectively, and for convenience, interpret $J(s, p)$ as the cost of solving the problem $p \in P$ with solver problem $s \in S$. Note that if $J(s, p)<J\left(s^{\prime}, p^{\prime}\right)$, then it can be said that $s \in S$ solves problem $p \in P$ better than solver $s^{\prime} \in S$ solves problem $p^{\prime} \in P$ (i.e., the problem $p \in P$ was easier for solver $s \in S$ than the problem $p^{\prime} \in P$ was for solver $s^{\prime} \in S$ ). 
For a given benchmarking context $\langle S, P, J\rangle$ assume further that the following assumptio,ns hold (where $n_{P}, n_{S}$ are given natural numbers):

$$
\left\{\begin{array}{cc}
(A 0) & P=\left\{1, \ldots, n_{P}\right\}, S=\left\{1, \ldots, n_{S}\right\} \\
(A 1) & J(s, p) \geq 0 \forall(s, p) \in S \times P \\
(A 2) & I_{P}=\sum_{p \in P} J(s, p)>0 \quad \forall s \in S
\end{array}\right.
$$

Assumption (A0) establishes that the sets $S, P$ are finite. Assumption (A2) can be interpreted as a no-triviality condition of the assessments (such that, as a requirement, each solver and each problem are to be tested with at least one problem and one solver, respectively). (A2) implies that $\left.I_{S P}=\sum_{s \in S, p \in P} J(s, p)\right\rangle 0$. The triple $\langle S, P, J\rangle$, which satisfies assumptions (A0), (A1), and (A2), is henceforth referred to as the benchmarking context.

Let us assume now that $\langle S, P, J\rangle$ is a given benchmarking context. Define the set of alternatives as $S$ i.e., $A=S=\left\{s_{1}, \ldots, s_{n_{S}}\right\}$. At the same time, define the criteria set $C=\left\{c_{1}, \ldots, c_{n_{P}}\right\}$ as follows: $c_{j}(\cdot)=J\left(\cdot, p_{j}\right): S \rightarrow \mathbb{R}$ for $p_{j} \in P, j=1, \ldots, n_{P}$; i.e., we can assume that $C=P$. Hence, we obtain decision matrix $X=\left[x_{i j}\right]$, where $x_{i j}=c_{j}\left(a_{i}\right)=$ $J\left(s_{i}, p_{j}\right), i=1, \ldots n_{S}, j=1, \ldots, n_{P}$. We assume also that $x_{j}^{\min }<x_{j}^{\max }$ for all $j=1, \ldots, n_{P}$. Now we can apply the game-theoretic approach for solving MODM problems, as it was described in the previous subsection.

\section{Benchmarking of Differential Evolution Algorithms}

We focus in this section on an illustrative example using the proposed method. To apply the proposed approach, we must solve the corresponding zero-sum game. To this end, we use the standard approach of reducing this game-theoretic problem to a linear programming problem. All calculations were performed using the MATLAB environment.

Note finally that, as we will see below, the results are quite appropriate and competitive and were found without any previous estimates of the importance of the criteria. For comparison, we also present here results obtained using the ENT method described above (see subsection 2.1).

The study [16] considered the 9 optimization algorithms and 25 test functions and correspondingly we consider the sets of 9 solvers and 50 problems (see Tables B.1- B4, Annex B.) The sources cited in these tables present detailed information on the selected algorithms and test functions. The ERTRSE metric/assessment function used in [16] (see Annex A for detail description).

Table B5, Annex B, presents the ERTRSE values for all problem-solver pairs. Obviously, assumptions (A0), (A1), and (A2) hold in the case under consideration, and hence, the benchmarking context $\langle S, P, J\rangle$ is fully determined.

Here we present the ranking results of the solvers/problems obtained using the proposed method, GTR, and, for comparison, the results obtained using the ENT method. First, note 
that the solution (equilibrium) in the mixed strategies for the corresponding zero-sum game is

$$
\begin{gathered}
\xi^{*}=(0.2299,0.2636,0,0,0,0.1976,0,0.3089,0) \\
\zeta^{*}=(0,0,0.2037,0,0,0,0.3000,0,0,0,0,0,0,0,0,0,0,0,0,0,0,0,0,0, \\
0,0,0,0,0,0,0,0,0,0,0,0,0,0,0,0.2960,0,0,0,0.2003,0,0,0,0,0,0)
\end{gathered}
$$

This solution can be interpreted as the distribution of some population according to preferences regarding solvers/problems (see subsection 2.2). Note also that using the proposed method, within a given benchmarking context, the solvers S01, S02, S06, S08 and the problems P03, P07, P40, P44 have a special status (are significant).

The results of the ranking of solvers and problems using the three methods indicated above are reported in Tables 1 and 2 and illustrated in Figures 1 and 2, respectively. Our calculations show that according to the GTR-ranking the solvers S01 and S09 (the problems P07 and P20) can be considered as "the first" and "the last", respectively, in the framework of the considered benchmarking context.

Table 1. Ranking solvers using different methods

\begin{tabular}{ccc}
\hline Solver & ENT & GTR \\
\hline S01 & 8 & 9 \\
S02 & 9 & 6 \\
S03 & 5 & 5 \\
S04 & 2 & 3 \\
S05 & 4 & 4 \\
S06 & 6 & 7 \\
S07 & 7 & 2 \\
S08 & 3 & 8 \\
S09 & 1 & 1 \\
\hline
\end{tabular}

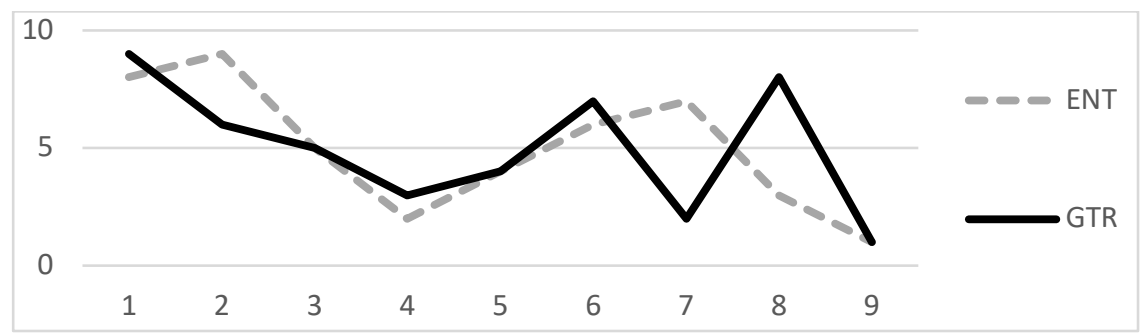

Figure 1. Comparison of rankings obtained using different methods.

Vertical axis-Rank, Horizontal axis-Solvers (see main text for explanation) 
Table 2. Ranking problems using different methods

\begin{tabular}{ccc|ccc}
\hline Problem & ENT & GTR & Problem & ENT & GTR \\
\hline P01 & 10 & 13 & P26 & 6 & 9 \\
P02 & 34 & 27 & P27 & 33 & 34 \\
P03 & 4 & 2 & P28 & 26 & 10 \\
P04 & 24 & 14 & P29 & 29 & 16 \\
P05 & 38 & 37 & P30 & 21 & 15 \\
P06 & 22 & 24 & P31 & 19 & 33 \\
P07 & 1 & 1 & P32 & 2 & 5 \\
P08 & 49 & 48 & P33 & 50 & 39 \\
P09 & 12 & 23 & P34 & 14 & 32 \\
P10 & 8 & 12 & P35 & 9 & 36 \\
P11 & 48 & 35 & P36 & 47 & 40 \\
P12 & 45 & 45 & P37 & 44 & 47 \\
P13 & 16 & 20 & P38 & 30 & 31 \\
P14 & 39 & 30 & P39 & 36 & 29 \\
P15 & 46 & 42 & P40 & 40 & 3 \\
P16 & 17 & 7 & P41 & 25 & 28 \\
P17 & 37 & 46 & P42 & 23 & 18 \\
P18 & 43 & 49 & P43 & 15 & 17 \\
P19 & 42 & 41 & P44 & 5 & 4 \\
P20 & 41 & 50 & P45 & 13 & 25 \\
P21 & 7 & 11 & P46 & 20 & 22 \\
P22 & 27 & 21 & P47 & 28 & 26 \\
P23 & 11 & 19 & P48 & 31 & 38 \\
P24 & 35 & 43 & P49 & 32 & 44 \\
P25 & 18 & 8 & P50 & 3 & 6 \\
\hline P19 & & & & & \\
\hline
\end{tabular}

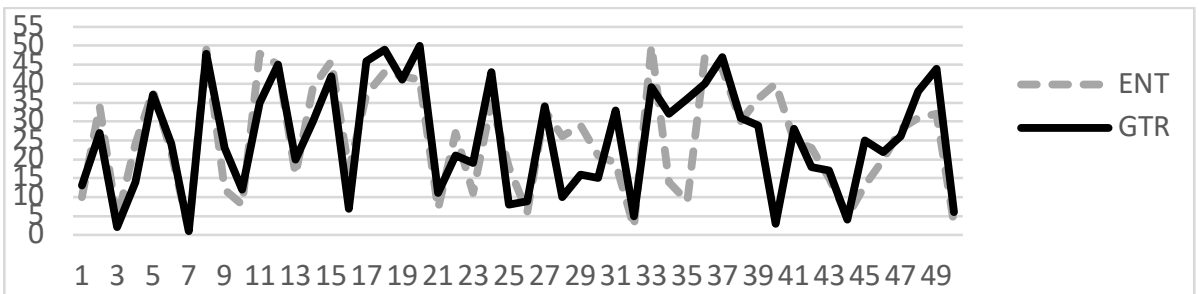

Figure 2. Comparison of rankings obtained using different methods Vertical axis-Rank, Horizontal axis-Problems (see main text for explanation) 


\section{Conclusion}

In this paper, we proposed a new method for benchmarking computational problems and their solvers. The method is based on a game-theoretic approach to the solving of MODM problems. The proposed method is sufficiently general to be used in other areas. Furthermore, we considered an illustrative example to demonstrate the viability and suitability of the proposed method for applications.

Note also that we only provided a tool for benchmarking a given set of solvers (problems) on a given set of problems (solvers) using a given performance metric, i.e., in the framework of the given benchmarking context. However, issues regarding selection of benchmarking context components remain open. The literature does not contain clear and direct recommendations regarding how to select a collection of solvers, problems, and performance metrics to obtain benchmarking results with proper justification. Hence, further development of benchmarking methods must focus to a greater extent on the justification of a benchmarking context's component choices.

\section{Annex A}

A description of the assessment function used in [16] follows. First, note that the expected running time (ERT), a widely used performance metric for optimization algorithms, is defined as

$$
\operatorname{ERT}(\tau)=\operatorname{mean}\left(M_{\tau}\right)+\frac{1-q}{q} N_{\text {max }}, q=\frac{N_{\text {succes }}}{N_{\text {total }}}
$$

where $\tau$ is a reference threshold value, $M_{\tau}$ is the number of function evaluations required to reach an objective value better than $\tau$ (such as successful runs), $N_{\max }$ is the maximum number of function evaluations per optimization run, $N_{\text {succes }}$ is the number of successful runs, $N_{\text {total }}$ is the total number of runs, and $q$ is the named success rate [1].

Note that ERT can be interpreted as the expected number of function evaluations of an algorithm to reach an objective function threshold for the first time and for the ERT performance measure, a threshold or success criterion is required. However, unlike conventional optimization problems (where ERT criterion is usually related to reaching the value of the known global optimum, within a specified tolerance), for difficult optimization problems the probability of coming close to the global optimum is negligible and a more acceptable alternative success criterion is required. Moreover, to compare qualitative performance using ERT for the difficult optimization problems, it is necessary that all compared algorithms meet the success criterion at least a few times. Correspondingly, in [16] was used the success criterion as reaching a target value which corresponds to the expected value of the best objective function value obtained from uniform random sampling (1000 samples). Next, for a test function $f$ determined the expected objective value $\mathrm{E}^{\mathrm{RSE}}(f)$, the estimation of which is based on the 100 repetitions. Finally, the ERT w.r.t. this objective function value limit was referred as ERTRSE (Random Sampling Equivalent-Expected Run Time) for the test function $f$. 


\section{Annex B}

Table B.1. Solvers

\begin{tabular}{|c|c|c|c|c|c|c|c|c|c|}
\hline Solver & S01 & S02 & S03 & S04 & S05 & S06 & S07 & S08 & S09 \\
\hline Algorithm & DE & DE2 & jDE & JADE & SADE & Code & epsDE & SQG & $\begin{array}{c}\text { SQG- } \\
\text { DE }\end{array}$ \\
\hline
\end{tabular}

Table B.2. Problems

\begin{tabular}{|c|c|c|c|c|c|}
\hline \multirow{2}{*}{ Problem } & \multicolumn{2}{|c|}{ Description } & \multirow{2}{*}{ Problem } & \multicolumn{2}{|c|}{ Description } \\
\hline & Dimension & Function & & Dimension & Function \\
\hline P01 & 30 & F01 & P26 & 50 & F01 \\
\hline P02 & 30 & F02 & P27 & 50 & F02 \\
\hline P03 & 30 & F03 & P28 & 50 & F03 \\
\hline P04 & 30 & F04 & P29 & 50 & F04 \\
\hline P05 & 30 & F05 & P30 & 50 & F05 \\
\hline P06 & 30 & F06 & P31 & 50 & F06 \\
\hline P07 & 30 & F07 & P32 & 50 & F07 \\
\hline P08 & 30 & F08 & P33 & 50 & F08 \\
\hline P09 & 30 & F09 & P34 & 50 & F09 \\
\hline P10 & 30 & F10 & P35 & 50 & F10 \\
\hline P11 & 30 & F11 & P36 & 50 & F11 \\
\hline P12 & 30 & F12 & P37 & 50 & F12 \\
\hline P13 & 30 & F13 & P38 & 50 & F13 \\
\hline P14 & 30 & F14 & P39 & 50 & F14 \\
\hline P15 & 30 & F15 & P40 & 50 & F15 \\
\hline P16 & 30 & F16 & P41 & 50 & F16 \\
\hline P17 & 30 & F17 & P42 & 50 & F17 \\
\hline P18 & 30 & F18 & P43 & 50 & F18 \\
\hline P19 & 30 & F19 & P44 & 50 & F19 \\
\hline P20 & 30 & F20 & P45 & 50 & F20 \\
\hline P21 & 30 & $\mathrm{~F} 21$ & P46 & 50 & F21 \\
\hline P22 & 30 & $\mathrm{~F} 22$ & P47 & 50 & F22 \\
\hline P23 & 30 & $\mathrm{~F} 23$ & P48 & 50 & F23 \\
\hline P24 & 30 & F24 & P49 & 50 & F24 \\
\hline P25 & 30 & $\mathrm{~F} 25$ & P50 & 50 & F25 \\
\hline
\end{tabular}


Table B.3. Test Functions

\begin{tabular}{|c|l|}
\hline Function & Short Description (see [23]) \\
\hline F01 & Shifted Sphere Function \\
F02 & Shifted Schwefel's Problem 1.2 \\
F03 & Shifted Rotated High Conditioned Elliptic Function \\
F04 & Shifted Schwefel's Problem 1.2 with noise in fitness function \\
F05 & Schwefel's Problem 2.6 with the global optimum on the bounds \\
F06 & Shifted Rosenbrock's Function \\
F07 & Shifted Rotated Griewank's Function \\
F08 & Shifted Rotated Ackley's Function with the global optimum on the bounds \\
F09 & Shifted Rastrigin's Function \\
F10 & Shifted Rotated Rastrigin's Function \\
F11 & Shifted Rotated Weierstrass Function \\
F12 & Schwefel's Problem 2.13 \\
F13 & Expanded Extended Griewank's plus Rosenbrock's Function \\
F14 & Shifted Rotated Expanded Scaffer's F6 \\
F15 & Hybrid Composition Function \\
F16 & Rotated Hybrid Composition Function \\
F17 & Rotated Hybrid Composition Function \\
F18 & Rotated Hybrid Composition Function \\
F19 & Rotated Hybrid Composition Functions with noise in fitness function \\
F20 & Rotated Hybrid Composition Function with a narrow basin for the global optimum \\
F21 & Rotated Hybrid Composition Function \\
F22 & Rotated Hybrid Composition Function with a high condition number matrix \\
F23 & Non-Continuous Rotated Hybrid Composition Function \\
F24 & Rotated Hybrid Composition Function \\
F25 & Rotated Hybrid Composition Function \\
\hline
\end{tabular}

Source: [16].

Table B.4. Algorithms

\begin{tabular}{|l|l|c|}
\hline \multicolumn{1}{|c|}{ Algorithm } & \multicolumn{1}{|c|}{ Short Description } & Source \\
\hline DE & "Rand/1/bin" Differential Evolution & {$[17]$} \\
DE2 & "Best/2/bin" Differential Evolution & {$[18]$} \\
jDE & Self-adapting Differential Evolution & {$[6]$} \\
JADE & Adaptive Differential Evolution & {$[23]$} \\
SaDE & Strategy adaptation Differential Evolution & {$[15]$} \\
Code & Composite trial vector strategy Differential Evolution & {$[21]$} \\
epsDE & Ensemble parameters Differential Evolution & {$[12]$} \\
SQG & Stochastic Quasi-Gradient search & {$[9]$} \\
SQG-DE & Stochastic Quasi-Gradient based Differential Evolution & {$[16]$} \\
\hline
\end{tabular}

Source: [16]. 
Table B5. ERTRSE Metric

\begin{tabular}{|c|c|c|c|c|c|c|c|c|c|}
\hline \multirow{2}{*}{ Problems } & \multicolumn{9}{|c|}{ Solvers } \\
\hline & S01 & S02 & S03 & S04 & S05 & S06 & S077 & S08 & S09 \\
\hline P01 & 11657 & 24049 & 491 & 264 & 329 & 904 & 606 & 202 & 168 \\
\hline P02 & 7401 & 11567 & 739 & 309 & 379 & 894 & 3653 & 137 & 318 \\
\hline P03 & 6536 & 32763 & 606 & 356 & 519 & 1148 & 1045 & 135 & 210 \\
\hline P04 & 6182 & 13326 & 570 & 285 & 342 & 662 & 1712 & 694 & 310 \\
\hline P05 & 4342 & 4766 & 536 & 311 & 527 & 871 & 564 & 459 & 160 \\
\hline P06 & 7404 & 11728 & 491 & 234 & 315 & 807 & 603 & 124 & 168 \\
\hline P07 & 256 & 124 & 482 & 310 & 596 & 879 & 169 & 32365 & 107 \\
\hline P08 & 2877 & 3011 & 1783 & 2084 & 2673 & 2720 & 2082 & 132 & 2771 \\
\hline P09 & 15735 & 24058 & 467 & 269 & 380 & 818 & 726 & 126 & 193 \\
\hline P10 & 11658 & 24054 & 474 & 256 & 320 & 850 & 630 & 201 & 172 \\
\hline P11 & 2414 & 1555 & 1967 & 1502 & 1321 & 1541 & 1979 & 368 & 1949 \\
\hline P12 & 1072 & 934 & 509 & 342 & 416 & 931 & 594 & 128 & 225 \\
\hline P13 & 15709 & 10150 & 524 & 224 & 231 & 723 & 898 & 161 & 289 \\
\hline P14 & 7497 & 19174 & 2735 & 1735 & 1509 & 2366 & 6379 & 6899 & 1658 \\
\hline P15 & 437 & 373 & 668 & 366 & 565 & 857 & 458 & 1016 & 185 \\
\hline P16 & 2999 & 11671 & 490 & 335 & 615 & 689 & 616 & 219 & 179 \\
\hline P17 & 6925 & 11670 & 514 & 362 & 572 & 845 & 693 & 9096 & 178 \\
\hline P18 & 1017 & 1036 & 501 & 314 & 460 & 741 & 341 & 263 & 143 \\
\hline P19 & 1271 & 1045 & 533 & 317 & 551 & 836 & 389 & 259 & 148 \\
\hline P20 & 1190 & 1098 & 491 & 315 & 545 & 1039 & 384 & 284 & 154 \\
\hline P21 & 9466 & 24251 & 498 & 253 & 327 & 901 & 597 & 301 & 175 \\
\hline P22 & 2433 & 2889 & 489 & 281 & 389 & 730 & 602 & 13320 & 202 \\
\hline P23 & 13650 & 24194 & 474 & 254 & 325 & 802 & 586 & 664 & 181 \\
\hline P24 & 7785 & 4449 & 544 & 272 & 376 & 911 & 573 & 10183 & 187 \\
\hline P25 & 220 & 115 & 445 & 304 & 554 & 855 & 167 & 5321 & 109 \\
\hline P26 & 19030 & 9054 & 439 & 205 & 254 & 674 & 579 & 192 & 178 \\
\hline P27 & 9055 & 8145 & 572 & 316 & 388 & 908 & 2135 & 127 & 296 \\
\hline P28 & 3819 & 10317 & 514 & 289 & 455 & 764 & 566 & 121 & 145 \\
\hline P29 & 19059 & 10175 & 538 & 341 & 434 & 920 & 2931 & 1332 & 310 \\
\hline P30 & 9173 & 19040 & 568 & 294 & 366 & 964 & 1418 & 451 & 214 \\
\hline P31 & 11557 & 13327 & 491 & 235 & 244 & 831 & 609 & 132 & 182 \\
\hline P32 & 394 & 187 & 443 & 289 & 536 & 884 & 231 & 24041 & 109 \\
\hline P33 & 1732 & 2347 & 2098 & 1792 & 2724 & 2522 & 2740 & 117 & 1960 \\
\hline P34 & 15719 & 19060 & 482 & 245 & 295 & 845 & 738 & 123 & 194 \\
\hline P35 & 24071 & 24059 & 456 & 235 & 306 & 739 & 661 & 169 & 188 \\
\hline P36 & 2024 & 2568 & 2187 & 1324 & 1814 & 1825 & 1675 & 347 & 1331 \\
\hline P37 & 1183 & 1263 & 560 & 340 & 418 & 1132 & 774 & 119 & 224 \\
\hline P38 & 7381 & 9057 & 514 & 227 & 193 & 604 & 1139 & 145 & 336 \\
\hline P39 & 7476 & 15824 & 1792 & 1310 & 1198 & 1296 & 3482 & 4706 & 1808 \\
\hline P40 & 341 & 291 & 557 & 354 & 556 & 852 & 331 & 205 & 173 \\
\hline P41 & 7506 & 11568 & 495 & 320 & 487 & 738 & 593 & 260 & 156 \\
\hline P42 & 11585 & 32369 & 640 & 327 & 515 & 794 & 809 & 5743 & 192 \\
\hline $\mathbf{P 4 3}$ & 10264 & 19135 & 631 & 319 & 380 & 1071 & 892 & 352 & 226 \\
\hline P44 & 24351 & 7387 & 511 & 290 & 389 & 866 & 728 & 307 & 210 \\
\hline P45 & 19307 & 13407 & 533 & 321 & 372 & 915 & 794 & 380 & 227 \\
\hline
\end{tabular}


(Table B5, continued)

\begin{tabular}{|c|c|c|c|c|c|c|c|c|c|c|}
\hline \multirow{2}{*}{ Problems } & \multicolumn{10}{|c|}{ Solvers } \\
\cline { 2 - 10 } & S01 & S02 & S03 & S04 & S05 & S06 & S077 & S08 & S09 \\
\hline P46 & 13571 & 9089 & 486 & 243 & 320 & 871 & 605 & 325 & 176 \\
P47 & 3311 & 5068 & 564 & 291 & 431 & 755 & 724 & 19048 & 153 \\
P48 & 7468 & 7544 & 483 & 248 & 333 & 880 & 590 & 537 & 170 \\
P49 & 15709 & 13335 & 498 & 231 & 245 & 784 & 688 & 8170 & 201 \\
P50 & 304 & 163 & 488 & 315 & 552 & 1038 & 210 & 13368 & 109 \\
\hline
\end{tabular}

Source: [16].

\section{References}

[1] Auger, A., Hansen, N., Performance evaluation of an advanced local search evolutionary algorithm, in: Proceedings of the IEEE Congress on Evolutionary Computation, 2, 2005, 1777-1784.

[2] Benson, H.Y., Shanno, D.F., Vanderbei, R.J., Interior-point methods for nonconvex nonlinear programming: Jamming and comparative numerical testing, Operations Research and Financial Engineering, Princeton University, Technical Report ORFE-0002, 2000.

[3] Billups, S.C., Dirkse, S.P., Ferris, M.C., A comparison of algorithms for large-scale mixed complementarity problems, Comput. Optim. Appl., 7,1997, 3-25.

[4] Bondarenko, A.S., Bortz, D.M., More, J.J., COPS: Large-scale nonlinearly constrained optimization problems, No. ANL/MCS-TM-237. Argonne National Lab., IL (US), 2000.

[5] Bongartz, I., Conn, A.R., Gould, N.I.M., Saunders, M.A., Toint, P.L., A numerical comparison between the LANCELOT and MINOS packages for large-scale numerical optimization, Report 97/13, Namur University, 1997

[6] Brest, J., Greiner, S., Boskovic, B., Mernik, M., Zumer, V., Self-adapting control parameters in differential evolution: A comparative study on numerical benchmark problems, IEEE transactions on evolutionary computation, 10, 6, 2006, 646-657.

[7] Conn, A.R., Gould, N.I.M., Toint, P.L, Numerical experiments with the LANCELOT package (Release A) for large-scale nonlinear optimization, Math. Program., 73, 1996, 73-110.

[8] Dolan, E.D., Moré, J.J., Benchmarking optimization software with performance profiles. Mathematical programming 91.2,2002, pp. 201-213.

[9] Ermoliev, Y. M., Methods of solution of nonlinear extremal problems, Cybernetics, 2,4, 1966, 1-14.

[10] Gogodze J., PageRank method for benchmarking computational Problems and their solvers, International Journal of Computer Science Issues, 15, 3, 2018, 1-7.

[11] Gogodze J., Using a Two-Person Zero-Sum Game to Solve a Decision-Making Problem, Pure and Applied Mathematics Journal, 7, 2, 2018, 11-19. 
[12] Mallipeddi, R., Suganthan, P. N., Pan, Q. K., Tasgetiren, M. F., Differential evolution algorithm with ensemble of parameters and mutation strategies, Applied Soft Computing, 11, 2, 2011, 1679-1696.

[13] Mittelmann, H., Benchmarking interior point LP/QP solvers, Optim. Methods Softw., 12, 1999, 655-670.

[14] Nash, S.G., Nocedal, J., A numerical study of the limited memory BFGS method and the truncated Newton method for large scale optimization, SIAM J. Optim., 1, 1991, $358-372$

[15]Qin, A. K., Huang, V. L., Suganthan, P. N., Differential evolution algorithm with strategy adaptation for global numerical optimization, IEEE transactions on Evolutionary Computation, 13,2, 2009, 398-417.

[16] Sala, R., Baldanzini N., Pierini M., SQG-Differential Evolution for difficult optimization problems under a tight function evaluation budget, in: International Workshop on Machine Learning, Optimization, and Big Data, Springer, Cham, 2017, 322-336.

[17] Storn, R., Price, K., Differential Evolution - a simple and efficient adaptive scheme for global optimization over continuous spaces, Technical Report TR-95-012, Berkeley: ICSI ,1995.

[18] Storn, R., Price, K., Differential evolution-a simple and efficient heuristic for global optimization over continuous spaces, J. of global optimization, 11,4, 1997, 341-359.

[19] Suganthan, P.N., Hansen, N., Liang, J.J., Deb, K., Chen, Y.P., Auger, A., Tiwari, S., Problem definitions and evaluation criteria for the CEC 2005 special session on realparameter optimization, KanGAL report, 2005005, 2005.

[20] Vanderbei, R.J., Shanno, D.F., An interior-point algorithm for nonconvex nonlinear programming, Comput. Optim. Appl., 13, 1999, 231-252.

[21] Wang, Y., Cai, Z., Zhang, Q., Differential evolution with composite trial vector generation strategies and control parameters, IEEE Transactions on Evolutionary Computation, 15,1, 2011, 55-66.

[22]Zeleny M., Multiple criteria decision making, New York: McGraw-Hill, 1982.

[23]Zhang, J., Sanderson, A. C., JADE: adaptive differential evolution with optional external archive, IEEE Transactions on evolutionary computation, 13,5, 2009, 945-958.

Received 18.07.2018, Accepted 14.01.2019 